\title{
Attitudes toward medication and the clinical variables in schizophrenia: Structural equation models
}

\author{
Alessandro Rossi ${ }^{1}$ \\ Roberta Pacifico' \\ Paolo Stratta ${ }^{2}$ \\ 'Department of Experimental \\ Medicine at University of L'Aquila, \\ Institute of Clinical Research \\ 'Villa Serena' Department of Clinical \\ Neuroscience, Città S. Angelo, \\ Italy; ${ }^{2}$ Department of Mental \\ Health, ASL 4, L'Aquila, Italy
}

This article was published in the following Dove Press journal:

Patient Preference and Adherence

27 August 2009

Number of times this article has been viewed

Objective: Our study uses data from a large clinical trial to further dissect the contributors attitude toward antipsychotic medication measured with the Drug Attitude Inventory (DAI) in schizophrenia.

Method: A total of 227 consecutive outpatients (140 males, 87 females) with schizophrenia or schizoaffective disorder according to DSM-IV criteria were evaluated. Structural equation models (SEM) were used to examine the relationship between attitudes toward medication as measured with the DAI and clinical variables assessed by Positive and Negative Syndrome Scale (PANSS) and Global Assessment of Functioning (GAF).

Results: Among four models, the data better fit a model in which attitudes toward medication (DAI-30) was predicted by PANSS Positive and Insight measures, and GAF (goodnessof-fit $=0.99$ )

Conclusion: The data best fit a model where the most complex interaction of insight measures and positive symptoms does predict attitudes toward medication.

Keywords: attitude toward medication, drug attitude inventory, structural equation models

\section{Introduction}

Schizophrenia is a chronic disease that requires long-term treatment. Adherence to the prescribed antipsychotic therapy is a crucial factor for a successful treatment outcome. ${ }^{1}$ Therefore, the patient's positive personal opinion towards the chosen treatment can facilitate a better compliance. . $^{2,3}$

Although a multitude of factors play a role in a patient's decision as to whether or not to take medication, attitudes and beliefs about health and illness have been consistently identified as the major factors in such decisions. These attitudes and beliefs are better understood in the context of the health-belief model (HBM). ${ }^{4,5}$ Many of the recent instruments that are often in use for measuring subjective responses and serve as predictors of adherence invariably include items that tap into specific components of the HBM that has been shown to influence adherence behavior. ${ }^{6-12}$

'Insight' is another factor frequently implicated as a mediator in the patient adherence to drug prescriptions. ${ }^{13,14}$ Sajatovic and colleagues ${ }^{15}$ studied insight into illness and attitudes towards medications (ATM) among in-patients with schizophrenia and found that the antipsychotic (AP) treatment improved symptoms and insight into illness, but did not appreciably change ATM. Adverse AP effects also are not consistently associated with negative attitude while insight to the illness is increasingly associated with negative attitude toward medication ${ }^{13,15}$ so that other variables are needed to explain patients' ATM.
Correspondence: Alessandro Rossi Department of Experimental Medicine, University of L'Aquila, Via Vetoio-Località Coppito II, 67 I00 L'Aquila, Italy

Tel +390859590267

Fax +390859590400

Email alessandro.rossi@cc.univaq.it 
The current report is an analysis of data of a large sample of subjects with schizophrenia eligible to a risperidone long-acting trial, ${ }^{16}$ aimed to further dissect the contributors of attitude toward antipsychotic medication measured with the Drug Attitude Inventory (DAI). Several studies have reported that a wide range of clinical, psychopathological, and demographic variables predict ATM, ${ }^{7-15,17}$ however, these observations mainly report that the worse measures (eg, severity of illness and insight) predicted a worse ATM. We further hypothesized that the same variables could have different load to predict attitude toward medication on the basis of different pattern of interaction among them (ie, from less to more complex interaction). With this aim, we decided to use the structural equation model (SEM) analysis to explore the best fit of prediction of selected clinical and psychopathological variables (ie, Positive and Negative Syndrome Scale [PANSS] scores and Global Assessment of Functioning [GAF]) with a priori variation of relationship among the set of predictors, as stated by the researchers on the basis of the literature. ${ }^{7,17}$

\section{Methods}

A total of 227 consecutive outpatients (140 male, 87 female, mean age $49.91 \pm 11.05$ standard deviation [SD], range 18-65) with schizophrenia or schizoaffective disorder according to the Diagnostic and Statistical Manual of Mental Disorders (DSM-IV) criteria, ${ }^{18}$ and who required long-term antipsychotic therapy were recruited. Diagnoses were made by a senior staff psychiatrist on the basis of clinical interview. At the time of recruitment, all patients were symptomatically stable and had been taking the same dose of antipsychotic agents for at least one month before the screening visit. Patients were considered stable if there had been no appreciable change in symptoms over the previous month, regardless of the severity of their symptoms. They were not considered to be either optimally treated or symptom-free. All these patients agreed to participate in a switching study to long-acting injectable risperidone and were effectively treated with long-acting risperidone. All patients or their legal representatives provided written informed consent to the study. Further information on this population is reported by Rossi and colleagues. ${ }^{16}$

\section{Psychopathological evaluation}

Symptom severity was assessed using the PANSS. ${ }^{19}$ Positive, negative, cognitive, and general psychopathology PANSS scores and 'insight' PANSS item were evaluated as reported by Daneluzzo and colleagues. ${ }^{20}$ Community functioning was evaluate using the GAF scale. ${ }^{21}$ Personal attitudes towards AP treatment was rated by a self-report questionnaire, the DAI-30 (see the Italian validation study for details). ${ }^{7,22}$ The DAI-30 is a valid and reliable tool used in clinical trials to investigate the patients' opinion towards therapy. All these evaluations were performed before patients switched to long-acting risperidone.

\section{Statistical analysis}

Structural equation models were used to examine the relationship between attitudes toward medication as measured with DAI and the clinical variables.

SEM is a statistical technique for testing and estimating causal relationships using a combination of statistical data and qualitative causal assumptions. The SEM is a statistical technique that combines elements of traditional multivariate models, such as regression analysis, factor analysis, and simultaneous equation modeling. ${ }^{23}$ The purpose is structure detection, ie, to examine the underlying (or latent) relationships between the variables. SEM extraction methods go one step further by adding the assumption that some of the variability in the data cannot be explained by the components (usually called factors in other extraction methods). As a result, the total variance explained by the solution is smaller; however, the addition of this structure to the factor model makes these methods ideal for examining relationships between the variables.

Since SEM is a confirmatory technique, the model must be specified correctly based on the type of analysis that the modeler is attempting to confirm. There are usually two main parts to SEM: the structural model showing potential causal dependencies between endogenous and exogenous variables and the measurement model showing the relations between the latent variables and their indicators. Parameter estimation is done by comparing the covariance matrices that represent the relationships between variables and the estimated covariance matrices of the best-fitting model. This is obtained through numerical maximization of a fit criterion as provided by maximum likelihood, weighted least squares, or asymptotically distribution-free methods. This is best accomplished by using a specialized SEM analysis program, SAS Proc CALIS (SAS Institute, Cary, NC).

In SAS Proc CALIS, when data fits the best model, the analysis will indicate minimum significant differences between the hypothesized model and the observed data. Model fit was assessed using the maximum-likelihood chi-squared test, the root mean square error of approximation (RMSEA), and by the comparison of the pair-wise correlation 
and covariance matrix with the estimates from the best-fit model.

The model we proposed in the study is based on the correlation matrix of three measures of psychotic symptoms (PANSS Positive, PANSS Negative, and PANSS General), two measures of insight (PANSS Item and PANSS Cognitive factors), one measure of GAF, and one measure of attitudes toward medication (DAI-30). We estimated this model by maximum likelihood, assessed its goodness-of-fit, and examined possible modifications of the model.

\section{Results}

Clinical features of the studied sample are reported in Table 1.

The hypothesized model 1 (Figure 1; Table 2 ), which assumes that all variables (PANSS Positive, PANSS Negative, PANSS General, PANSS Cognitive, PANSS G12, and GAF) are correlated with attitudes toward medication (DAI-30) had a satisfactory goodness-of-fit model. The goodness-of-fit statistics indicate the appropriateness of the linear structural equation model for the given dataset (covariance matrix; Goodness-of-Fit Index [GFI] $=0.68$, maximum likelihood estimation fit function $=2.97$, RMSEA estimate $=0.99$ ).

The hypothesized model 2 (Figure 1; Table 2), which assumes that PANSS variables but not GAF are correlated with attitudes toward medication (DAI-30) also had a satisfactory goodness-of-fit model (GFI =0.61, maximum likelihood estimation fit function $=3.43$, RMSEA estimate $=1.31$ ).

The hypothesized model 3 (Figure 1; Table 2), which assumes that all variables (PANSS Positive, PANSS Negative, PANSS General, PANSS Cognitive, PANSS Insight, and GAF) are correlated with attitudes toward medication

Table I Clinical features of the studied sample $(n=227)$

\begin{tabular}{|c|c|c|c|}
\hline \multirow[t]{2}{*}{ Variable } & \multicolumn{3}{|l|}{ Score } \\
\hline & Possible range & Mean (SD) & Range \\
\hline PANSS - Positive & 7 to 49 & $17.95(6.01)$ & $7-38$ \\
\hline PANSS - Negative & 7 to 49 & $25.10(7.82)$ & $7-45$ \\
\hline PANSS - General & 16 to 112 & $45.19(11.83)$ & 19-76 \\
\hline $\begin{array}{l}\text { PANSS - Cognitive factors } \\
\text { (CF) P2 + N5 + N7 + G4 + } \\
\text { G5 + GII + GI2 }\end{array}$ & 7 to 49 & $21.44(6.72)$ & $7-40$ \\
\hline PANSS - Item GI2 & I to 7 & $3.23(1.50)$ & $\mathrm{I}-7$ \\
\hline DAl-30 & 25 to 50 & $42.00(5.85)$ & $26-50$ \\
\hline GAF & 0 to 100 & 48.83 (I I.22) & $21-76$ \\
\hline
\end{tabular}

Abbreviations: DAI-30, Drug Attitude Inventory; GAF, Global Assessment of Functioning; PANSS, Positive and Negative Syndrome scale.
(DAI-30) had a similar satisfactory goodness-of-fit model $(\mathrm{GFI}=0.76$, maximum likelihood estimation fit function $=3.49$, RMSEA estimate $=0.83$ ). In comparison to model 1, we added a possible relationship among the variables group "PANSS Positive, PANSS Negative, PANSS General" and among the variables group "PANSS Cognitive, PANSS Insight".

The hypothesized model 4 assumes that the variables PANSS Positive, PANSS G12 and GAF are correlated with attitudes toward medication (DAI-30) (Figure 1; Table 2). In this model we added a possible relationship among the variables group "PANSS Positive, PANSS Negative, PANSS General" and among the variables group "PANSS Cognitive, PANSS Insight". We observed a substantial increase in the GFI, a reduction in the maximum likelihood chi-squared test, and a reduction in the RMSEA, indicating a better model fit for model 4 compared with the previous models (GFI $=0.99$, maximum likelihood estimation fit function $=0.005$, RMSEA estimate $=0.02$ ).

The maximum likelihood function is a function which reflects the difference between the observed and predicted by the model, covariance matrices. The more complex the model, the more likely a good fit. In a just-identified model, with as many parameters as possible while still achieving a solution, there will be a perfect fit. In comparison to the complexity of the proposed model 4 where the three PANSS subscales are singly considered and introduced in the model as single explanatory variables, the low sample size could be identified as a possible confounder in the reproducibility of the model.

\section{Discussion}

The issue of attitude toward medication in schizophrenia has gained increasing attention both for psychopathological and pharmacotherapeutic reasons. ${ }^{24}$ Among the first is the concept that insight and awareness of symptoms could positively predict drug attitude and ultimately outcome. ${ }^{14,15,17}$ Among the latter, generally from clinical trials, there are data that switching to newer antipsychotics could favor better attitude and ultimately better compliance. ${ }^{25,26}$ Since their original description in the self-report of their experience of neuroleptic treatment, the attitude of schizophrenic patients toward their pharmacologic treatment was believed to predict drug compliance. ${ }^{2,7}$

Because several factors could predict drug attitude to medication, we followed the model proposed by Day and colleagues ${ }^{12}$ to use SEM to explore the relationship between clinical variables and DAI in a large sample of 

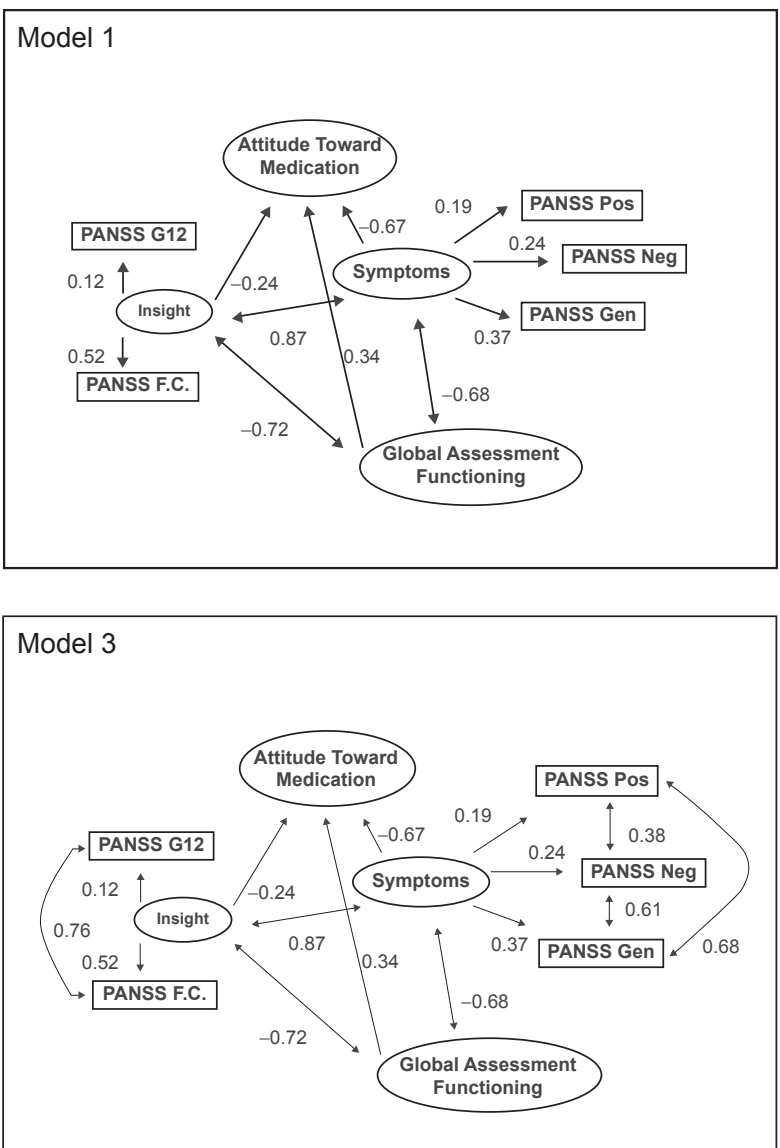

Model 2

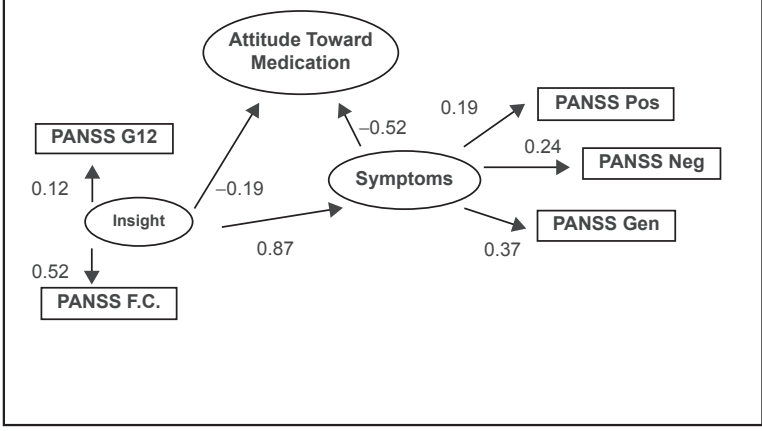

Model 4

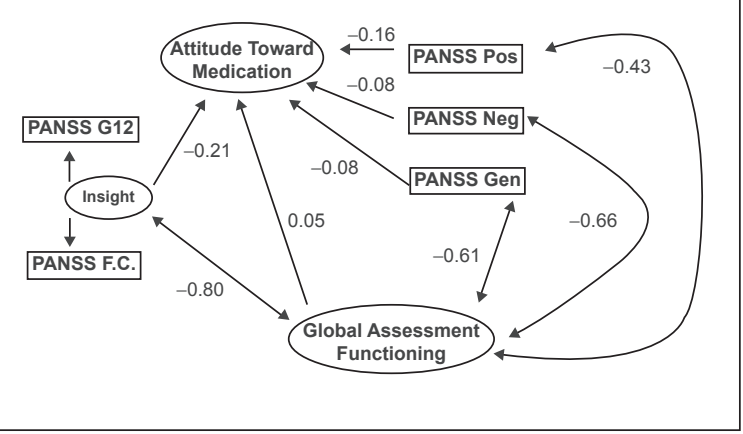

Figure I Hypothesized models with fitted coefficients.

Notes: The arrows represent the linear equation relationships and it is used to indicate causal flow from one variable to another (direct effects). The arrows with two points represent the correlations between groups of variables.

patients with schizophrenia and schizoaffective disorders. We used different SEM models, each differing on the basis of an a priori hypothesis of the authors about relationship between PANNS factors and $\mathrm{GAF}^{19,26}$ that offer different clinical considerations. Model 3 assessed GAF separately from PANSS factors and ruled out a nontrivial contribution of GAF itself (ie, better GAF means a better attitude toward medication). When GAF was believed to interact with
PANSS factors (model 4), PANSS Positive gained more attention. As expected insight-cognition does have a higher impact on DAI-30 after controlling GAF.

We appreciate that attitude toward medication is a complex and multifactorial construct depending on its assessment and psychosocial variables as recently reported by Day and colleagues. ${ }^{12}$ Nevertheless, symptom dimensions do have a crucial role. Depending on the a priori hypothesis on

Table 2 Structural equation modeling: Hypothesized models with fitted coefficients

\begin{tabular}{|c|c|c|c|c|}
\hline Variables in the model & GFI' & $\begin{array}{l}\text { Maximum } \\
\text { likelihood } \\
\text { estimation }\end{array}$ & $\begin{array}{l}\text { Model } \\
\text { chi-squared } \\
P\end{array}$ & $\begin{array}{l}\text { RMSEA }^{2} \\
(90 \% \mathrm{CL})\end{array}$ \\
\hline I. PANSS and GAF & 0.68 & 2.97 & $<0.000$ I & $0.99(0.93-1.06)$ \\
\hline 2. PANSS, but not GAF & 0.61 & 3.43 & $<0.000$ I & 1.31 (I.23-1.39) \\
\hline $\begin{array}{l}\text { 3. PANSS and GAF with relationship within groups } \\
\text { of variables }\end{array}$ & 0.76 & 3.49 & $<0.0001$ & $0.83(0.78-0.88)$ \\
\hline $\begin{array}{l}\text { 4. PANSS, GAF and insight (PANSS GI2 and CF) with } \\
\text { relationship between groups of variables }\end{array}$ & 0.99 & 0.005 & $=0.298$ & $0.02(0-0.18)$ \\
\hline
\end{tabular}

Abbreviations: CF, ; CL, ; GAF, Global Assessment of Functioning; GFI, Goodness-of-fit Index; PANSS, Positive and Negative Syndrome scale; RMSEA, root mean square error. 
the direction of causal link between the assessed variables, the available data could fit with different models. Even though the variables we selected do have a clear impact in determining ATM, we show that the most complex data set does have higher GFI in predicting ATM, which demonstrates the inherent complexity of the construct. The lack of systematic assessment of drug-related side-effects and other qualities of life-related variables is a limitation of this study. ${ }^{27} \mathrm{~A}$ deeper psychopathological and psychosocial analysis could better explain the meaning of ATM. This could add more information toward intervention aimed to increase compliance. ${ }^{28}$

\section{Acknowledgments}

The authors wish to thanks Janssen-Cilag Italy for permision to access this study Data Base and Dr Giorgio Reggiardo for his statistical assistance. The authors report no conflicts of interest in this work.

\section{References}

1. Lehman AF, Lieberman JA, Dixon LB, et al; American Psychiatric Association; Steering Committee on Practice Guidelines. Practice guideline for the treatment of patients with schizophrenia. Second edition. Am J Psychiatry. 2004;161(2 Suppl):1-56.

2. Awad AG. Subjective response to neuroleptics in schizophrenia. Schizophr Bull. 1993;19:609-618.

3. Awad AG, Voruganti LNP, Heslegrave RJ. The aims of antipsychotic medications: what are they and are they being achieved? CNS Drugs. 1995;4:8-16

4. Rosenstock IM. The health belief model and preventive health behaviour. Health Educ Monogr. 1974;2:354-383.

5. Bebbington PE. The content and context of compliance. Int Clin Psychopharmacol. 1995;9(Suppl 5):41-50.

6. Awad AG, Voruganti LNP, Heslegrave RJ, Hogan TP. Assessment of the patient's subjective experience in acute neuroleptic treatment: implications for compliance and outcome. Int Clin Psychopharmacol. 1996;11(Suppl 2):55-60.

7. Hogan TP, Awad AG, Eastwood R. A self-report scale predictive of drug compliance in schizophrenics: reliability and discriminative validity. Psychol Med. 1983;13:177-183.

8. Weiden P, Rapkin B, Mott T, et al. Rating of medications influences (ROMI) scale in schizophrenia. Schizophr Bull. 1994;20:297-310.

9. Naber DA. A self-rating scale to measure subjective effects of neuroleptic drugs, relationship to objective psychopathology, quality of life, compliance and other clinical variables. Int Clin Psychopharmacol. 1995;10(Suppl 3):133-138.

10. Voruganti LNP, Awad AG. Personal evaluation of transitions in treatment (PETiT): a scale to measure subjective aspects of antipsychotic drug therapy in schizophrenia. Schizophr Res. 2002;56:37-46.
11. Hofer A, Kemmler G, Eder U, Honeder M, Hummer M, Fleischhacker WW. Attitudes toward antipsychotics among outpatient clinic attendees with schizophrenia. J Clin Psychiatry. 2002;63(1):49-53.

12. Day J, Bentall RP, Roberts C, et al. Attitudes toward antipsychotic medication. the impact of clinical variables and relationships with health professionals. Arch Gen Psychiatry. 2005;62:717-724.

13. Garavan J, Browne S, Gervin M, Lane A, Larkin C, O'Callaghan E. Compliance with neuroleptic medication in outpatients with schizophrenia; relationship to subjective response to neuroleptics; attitudes to medication and Insight. Compr Psychiatry. 1998;39:215-219.

14. Kemp R, David A. Psychological predictors of insight and compliance in psychotic patients. Br J Psychiatry. 1996;169:444-450.

15. Sajatovic M, Rosch DS, Sivec HJ, et al. Insight into illness and attitudes toward medications among inpatients with schizophrenia. Psychiatr Serv. 2002;53:1319-1321.

16. Rossi A, Bagalà A, Del Curatolo V, et al. Remission in schizophrenia: one-year Italian study of risperidone long-acting injectable (RLAI) in patients with schizophrenia or schizoaffective disorder. Hum Psychopharmacol (submitted).

17. Freudenreich O, Cather C, Evins AE, Henderson DC, Goff DC Attitudes of schizophrenia outpatients toward psychiatric medications: relationship to clinical variables and insight. J Clin Psychiatry. 2004;65:1372-1376.

18. American Psychiatric Association. Diagnostic and Statistical Manual of Mental Disorders (4th. edition revised). American Psychiatric Association: Washington DC; 1994.

19. Kay SR. Positive and Negative Syndromes in Schizophrenia: Assessment and Research (Clinical and Experimental Psychiatry). New York, NY: Brunner-Mazel Inc; 1991.

20. Daneluzzo E, Arduini L, Rinaldi O, et al. PANSS factors and scores in schizophrenic and bipolar disorders during an index acute episode: a further analysis of the cognitive component. Schizophr Res. 2002; 56:129-136

21. Endicott J, Spitzer RL, Fleiss JL, Cohen J. The Global Assessment Scale: A procedure for measuring overall severity of psychiatric disturbance. Arch Gen Psychiatry. 1976;33:766-771.

22. Rossi A, Arduini L, De Cataldo S, Stratta P. Gli aspetti soggettivi del trattamento con farmaci antipsicotici: studio di validazione della versione italiana della Drug Attitude Inventory (DAI). Epidemiol Psichiatr Soc. 2001;10:107-113.

23. Kaplan, DW. Structural Equation Modeling: Foundations and Extensions. SAGE Publications: California; 2000.

24. Hofer A, Fleischhacker W. Attitudes towards medication in patients with schizophrenia. Acta Psychiatr Scand. 2006;113(3):161-162.

25. Awad G, Voruganti L. New antipsychotics, compliance, quality of life, and subjective tolerability. Are patients better off? Can J Psychiatry. 2004;49:297-302.

26. Goldman HH, Skodol AE, Lave TR. Revising axis V for DSM-IV: a review of measures of social functioning. Am J Psychiatry. 1992;149: $1148-1156$

27. Bebbington PE, Angenermyer M, Azorin JM, Marwaha S, Marteau F, Toumi M. Side-effects of antipsychotic medication and health-related quality of life in schizophrenia. Acta Psychiatr Scand. 2009; 119( Suppl 438):22-28.

28. Zygmunt A, Olfson M, Boyer CA, Mechanic D: Intervention to improve adherence in schizophrenia. Am J Psychiatry. 2002;159:1653-1664.
Patient Preference and Adherence

\section{Publish your work in this journal}

Patient Preference and Adherence is an international, peer-reviewed, open access journal that focusing on the growing importance of patient preference and adherence throughout the therapeutic continuum. Patient satisfaction, acceptability, quality of life, compliance, persistence and their role in developing new therapeutic modalities and compounds to

\section{Dovepress}

optimize clinical outcomes for existing disease states are major areas of interest. This journal has been accepted for indexing on PubMed Central. The manuscript management system is completely online and includes a very quick and fair peer-review system. Visit http://www.dovepress.com testimonials.php to read real quotes from published authors. 